\title{
Transverse aeroelastic instability of guard cables with beacons excited by a longitudinal wind
}

\author{
Omar Gómez-Ortega a ${ }^{\mathrm{a}}$, Marta Landeira ${ }^{\mathrm{b}}$, Mikel Ogueta-Gutiérrez ${ }^{\mathrm{a}}$, \\ Sebastián Franchini ${ }^{a}$, Angel Sanz-Andres ${ }^{a, *}$, Marcos Chimeno ${ }^{a}$, \\ Elena Roibás-Millán ${ }^{a}$, Andres Garcia-Perez ${ }^{\text {a }}$ \\ a IDR/UPM, Universidad Politécnica de Madrid, Pza. del Cardenal Cisneros 3, 28040 Madrid, Spain

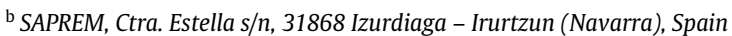

\begin{abstract}
A B S T R A C T
Guard cables, also named ground cables, protect the overhead power lines from electrical discharges that occur during storms. They are installed above the power conductor and therefore, they are the highest cable installed. In some places they are provided with beacon spheres that are visible indicators used for identifying the overhead power lines, to warn low flying airplanes and helicopters of the obstructions. A new transverse aeroelastic instability that appears in guard cables with beacons when excited by a longitudinal wind (TILoW for short) has recently been reported to us. In some cases, a vertical or horizontal oscillatory motion takes place, whereas in other cases the beacon centers follow circular orbits placed also in the transverse plane, while each beacon follows the motion but remaining parallel to itself. The centers of the orbits seem to be close to the static equilibrium position of the guard cable catenary. This whirling motion can be identified as a kind of autorotation. In this work the instability is described, and vortex induced vibration (VIV) is suggested as a candidate responsible for the aerodynamic force exciting the oscillation modes. For the whirling (also could be denoted "revolving") mode a parallel investigation is ongoing. In order to clarify the problem, a model of the elastic configuration that is excited by the aerodynamic loads is analyzed, the eigenmodes and eigenvalues are presented, and a preliminary order of magnitude assessment of the field data is performed. Frequencies and mode shapes derived from the elastic model and those from the video sequences are found to be in agreement. The oscillation results are compatible with the existence of an alternating force produced by vortex shedding from the sphere. Unfortunately, the assessment could not be pursued further due to the absence of data on the wind speed during the reported sequences.

A simplified model of the cable with only one beacon (a swinging sphere) has been tested in a low speed wind tunnel, and the main modes (VIV oscillation and whirling autorotation) have been reproduced and characterized.
\end{abstract}

* Corresponding author.

E-mail address: angel.sanz.andres@upm.es (A. Sanz-Andres). 


\begin{tabular}{|c|c|}
\hline \multicolumn{2}{|c|}{ Nomenclature } \\
\hline$A_{y}^{*}$ & Dimensionless amplitude \\
\hline$d_{B}$ & Beacon diameter \\
\hline$D_{N R}$ & Matrix of the dynamics \\
\hline$f$ & Circular frequency \\
\hline$f_{0}$ & Reference circular frequency \\
\hline$f_{e}$ & Reported frequency \\
\hline$f_{g}$ & Frequency for minimum rotation speed \\
\hline$f_{R}$ & Natural frequency \\
\hline$f_{v o}$ & Vortex shedding frequency \\
\hline$f^{*}$ & Dimensionless frequency \\
\hline$g$ & Gravity acceleration \\
\hline$I_{u}$ & Longitudinal turbulence intensity \\
\hline$k$ & Stiffness \\
\hline$l_{R}$ & Beacon separation distance \\
\hline$l_{S}$ & Distance between end cable supports, span length \\
\hline$L_{u}$ & Integral length of longitudinal turbulence \\
\hline$m$ & Point mass \\
\hline$m_{B}$ & Beacon mass \\
\hline$m_{d}$ & Mass of the displaced fluid \\
\hline$m_{e}$ & Equivalent mass \\
\hline$m^{*}$ & Reduced mass \\
\hline$N_{M}$ & Mode number \\
\hline$N_{R}$ & Number of beacons \\
\hline$n$ & Characteristic frequency of the wind turbulence fluctuation $u$ \\
\hline$q_{i}$ & Point mass displacement \\
\hline$q_{0 i}$ & Complex amplitude \\
\hline $\operatorname{Re}$ & Reynolds number \\
\hline$r_{g}$ & Pendulum length for oscillations, revolving radius for whirling motion \\
\hline$r_{m}$ & Participation coefficient \\
\hline St & Strouhal number \\
\hline$S_{u}$ & Spectral density of the turbulent fluctuation $u$ \\
\hline$S_{y}$ & Spectral density of the displacement \\
\hline$t$ & Time \\
\hline$T$ & Kinetic energy \\
\hline$T_{0}$ & Tension \\
\hline$U$ & Potential energy \\
\hline$U_{\infty}$ & Incoming flow speed \\
\hline$U_{\infty 0}$ & Reference wind speed \\
\hline$U^{*}$ & Reduced wind speed \\
\hline$u$ & Turbulent fluctuation of $U_{\infty}$ \\
\hline$y_{\text {rms }}$ & Root mean square value of $y(t)$ \\
\hline$x$ & Longitudinal position \\
\hline$X$ & Dimensionless eigenvalue \\
\hline$\beta$ & Cable-wind angle \\
\hline$\omega$ & Angular frequency \\
\hline$\omega_{\lim }$ & Limit value for a large number of beacons \\
\hline$\omega_{\mathrm{g}}$ & Angular frequency for gravity compensation \\
\hline$\omega_{0}$ & Reference angular frequency \\
\hline$\rho_{c}$ & Cable density (mass per unit length) \\
\hline$\sigma_{u}$ & Standard deviation of $u$ \\
\hline
\end{tabular}




\section{Introduction}

Guard cables, also known as ground cables, fulfill the function of protecting the overhead power lines from electrical discharges that occur during storms. They are installed above the power conductor, and therefore, they are the highest cables installed (Fig. 1). The beacon spheres are a type of Aircraft Warning Markers that are visible indicators used for the identification of tall structures, such as overhead power lines, as well as to warn low flying airplanes and helicopters of obstructions. In most jurisdictions, they are legally mandated for public safety, and must follow the international recommendations given by ICAO (International Civil Aviation Organization) or FAA (Federal Aviation Authority). The guard cables provided with beacon spheres are very often placed near airports where the airplanes are approaching or departing, and so are closer to the ground and consequently in danger of interfering with the power lines. Other locations are near rivers, lakes or fjord crossings, which are often located in deep valleys, and serve as well marked routes for general aviation aircraft.

The aim of this paper is to report on an apparently new instability, which affects guard cables with beacons. It occurs when the wind approaches in the direction of the power line (near zero cable angles). In some cases, the beacons showed oscillations in vertical and/or horizontal directions, while in other cases a kind of whirling autorotation appeared, always in the transverse plane to the guard cable. It seems that this instability has not been reported yet because it does not appear very often.

Wind effects (sometimes combined with ice, snow, rain) produce aeroelastic instabilities in overhead high voltage transmission lines (galloping, vortex-induced vibrations), when the wind direction is close to a normal incidence. A large number of research reports have been published on this subject. A fairly complete review can be found in EPRI (2009).

In a first approach, one of the possible candidate phenomena considered responsible for the aerodynamic loading leading to instability was galloping in one of its several forms. In this regard, different types of galloping of overhead transmission lines or other long span cables were reported and analyzed experimentally or theoretically by Davis et al. (1963), Simpson (1965), Dale et al. (1968), Bartoli et al. (2006), Macdonald and Larose (2006), Macdonald et al. (2008), Hung and Yamaguchi (2014) and Zhou and Liu (2015).

Sectional studies to analyze the galloping instability and wake induced vibrations of smooth cylinders, stranded or bundle sections on a free stream or in the wake of another body are reported by Wardlaw et al. (1975), King (1976), Zdravkovich (1977), Ramberg (1978), Wong (1980), Ruscheweyh (1983), Shiraishi (1986), Horton et al. (1988), Knisely and Kawagoe (1990), Batill et al. (1989), Batill and Nebres (1992), Jones (1992), Nebres et al. (1993); Nebres and Batill (1993), Smith (1993), Cigada et al. (1997), Chabart and Lilien (1998), Eguchi et al. (2002), Carassale et al. (2005), Cheng and Tanaka (2005), Macdonald and Larose (2008a,b) and Assi et al. (2010).

The above-mentioned papers deal with cables (without beacons) blown by a cross wind. However, the TILoW instability has been observed only in guard cables with a significant number of beacons placed at a longitudinal wind incidence. This fact suggests the need to pay attention to the aerodynamic loading on the beacons. In this regard, the works reported on both elastically supported and tethered spheres, in uniform or non-uniform flows, from Auton (1987), Williamson and Govardhan (1997), Moraga et al. (1999), Jauvtis et al. (2001), Govardhan and Williamson (2005), and Williamson and Govardhan (2008) should be considered.

In the case of tethered spheres, the flow direction did not coincide with the cable direction, as the sphere is heavier or lighter than the fluid. Besides, the action of gravity introduces a new direction that breaks the axial symmetry in the streamwise direction, so that the trajectory of oscillations of the tethered sphere takes a symmetric shape around the vertical plane that passes through the cable anchoring point and contains the flow direction (Williamson and Govardhan, 2008).

Special attention should be paid to the work of Provansal et al. (2004) on the motion of a longitudinally blown tethered sphere (conical pendulum). In this case, the direction of gravity coincides with the flow direction (the experiment is performed in a vertical water channel), and the axial symmetry is maintained. The observed trajectory of the sphere depended on the initial conditions: elliptic, quasi-circular, even planar oscillations may occur. Planar oscillations were unstable, eventually leading to circular or elliptic trajectories (conical pendulum in autorotation). The type of motions reported for the longitudinally blown tethered sphere can be considered as the most similar to the observed beacon motion in a guard cable under TILoW instability. However, there are two important differences: in a guard-cable the beacon is fixed to the cable at both ends and it is not isolated, it is a multibody system. In both cases, the effect of gravity is partially compensated by the cable tension.

The idea of this paper is to present the field data and to describe the TILoW instability (Section 2) aiming to find some candidate phenomena that could cause it. To support this study, the elastic problem of a cable fitted with several masses (which represent the beacons) is formulated, and the eigenvalues and associated eigenvectors are determined (Section 3). It can be shown that the motion derived from the eigenvector shapes is compatible with the motion observed in the field (Section 4). Therefore, an appropriate aerodynamic loading could excite one of these eigenmodes (Section 5). The results of wind tunnel tests on a simplified configuration are presented in Section 6. Finally, conclusions are drawn in Section 7.

\section{Field data}

The source of the information on this instability is a set of videos obtained by casual observers. From the analysis of these video images ( 25 frames per second), it is possible in some cases to determine the whirling rate or oscillation frequency of the beacons, and the mode shape (qualitatively). Several sources of information have been considered, denoted as Case- 1 , Case- 2 and Case -3 , among others, and are presented in the following paragraphs. The analysis of the videos was performed using a commercial video editor. The accuracy of the time tagging was $0.0335 \mathrm{~s}$ (one video frame interval). 
Table 1

Characteristics of the instability in Case-2 location. Oscillation in vertical direction.

\begin{tabular}{llllll}
\hline Vídeo WA & Perspective & Period $[\mathrm{s}]$ & Frequency $[\mathrm{Hz}]$ & Mode (Fig. 3b) & Beacons on scene (out of 15) \\
\hline 0024 & Along line & 0.567 & 1.76 & A (up) & 5 (Fig. 3a left) \\
0025 & Slanted & 0.700 & 1.43 & B (down) & 6 (Fig. 3a right) \\
\hline
\end{tabular}

\subsection{Case-1}

Some pictures obtained from a video taken in South America, denoted Case-1, are shown in Figs. 1 and 2. The guard cable has five beacons $\left(N_{R}=5\right)$. The motion evolution is obtained by comparing several consecutive frames of the video. The sequence of positions of each beacon defines a circular (or quasi-circular) orbit around the equilibrium position (catenary shape), with an angular speed of $1.50 \mathrm{~Hz}$. The observed motion is a transverse whirling. Interestingly, the shape of the instantaneous deformation of the cable with regard to the catenary shape is quite similar to the highest deformation mode ( $N_{M}=N_{R}=5$, where $N_{M}$ is the number of mode) of the oscillatory motion, which consists of an alternative displacement of the beacons in opposite direction. This motion can be described as a collective whirling autorotation (see Section 3).

\subsection{Case-2}

Some of the videos analyzed (denoted as WA0024 and WA0025) were taken of a transmission line in another South American country, denoted as Case-2. The conclusions obtained from the video sequence analysis are summarized in Table 1 , and some frames are presented in Fig. 3a. In this case the observed motion is a vertical oscillation.

Unfortunately, it is not possible to determine the complete mode shapes, as only a part of the whole span (a few wavelengths) is in the field of view, only 5 beacons (out of 15) in video WA0024 and 6 beacons in video WA0026. In some frames it is also difficult to determine the motion. The visible parts of the mode shapes have been obtained from video sequences and are sketched in Fig. 3b. The amplitude shown is only qualitative (it amounts to a fraction of the beacon diameter), in order to identify the mode shape. As shown in Table 1, both the mode shapes and frequencies are different in these two videos, the higher the deformation mode, the higher the mode frequency (see Table 4).

\subsection{Others}

Two other cases have been reported to the authors. From the former, a picture obtained from a video taken in another location in South America, denoted Case-3, is shown in Fig. 4.

The total number of beacons is not available in this case. The observed motion is also a transverse whirling in the highest deformation mode (alternative displacement). The only information that can be extracted is the rotation frequency ( $11 \mathrm{rev} / 7$ $\mathrm{s}=1.57 \mathrm{~Hz})$.

From another source ( private communication PC), data of a cable that contains 9 beacons has been obtained. The revolving rate of all beacons was the same, and the revolving radius of the beacon center changed depending on the beacon position along the cable, from $0.2 d_{B}$ to $0.6 d_{B}\left(d_{B}\right.$ being the beacon diameter, $\left.d_{B} \cong 0.6 \mathrm{~m}\right)$. From additional information, it was deduced that the relative wind direction coincided with the line direction. The observed motion was also a transverse whirling in the highest deformation mode (alternative displacement) with an angular speed of $1.50 \mathrm{~Hz}$, approximately.

\subsection{Comments on field data}

To the authors' knowledge, the TILoW phenomenon has not been reported for guard cables with few beacons, even though it has been the most common configuration for years. In fact, this instability has been reported only recently for guard cables with quite a large number of beacons. This number has recently been increased due to a change in the implementation of the regulations (ICAO, 2009). The distance between contiguous beacons must be less than $30 \mathrm{~m}$, but the number of beacons has been increased from typically 3 to 5 beacons placed close to the line span center (and only just above the road to be marked), to cover now the full span length (more than 15 beacons for span lengths larger than $450 \mathrm{~m}$ ).

\section{Elastic problem. Mathematic model}

Before starting to analyze the aerodynamic phenomena that can be responsible for this motion, the results of the elastic problem are presented in order to analyze the compatibility of the elastic displacement eigenmodes with the observed motion.

A simple mathematical model of the guard cable (with beacons) is presented in this section. According to the parameters of the guard cables, a shallow catenary approximation is considered. The transverse whirling motion of the beacon center around the catenary equilibrium shape can also be considered as a coupled oscillation in both horizontal and vertical directions with the same frequency and amplitude. Therefore, the results obtained on mode shapes and eigenfrequencies are applicable to these three motions (vertical and horizontal oscillations, and whirling autorotation). 


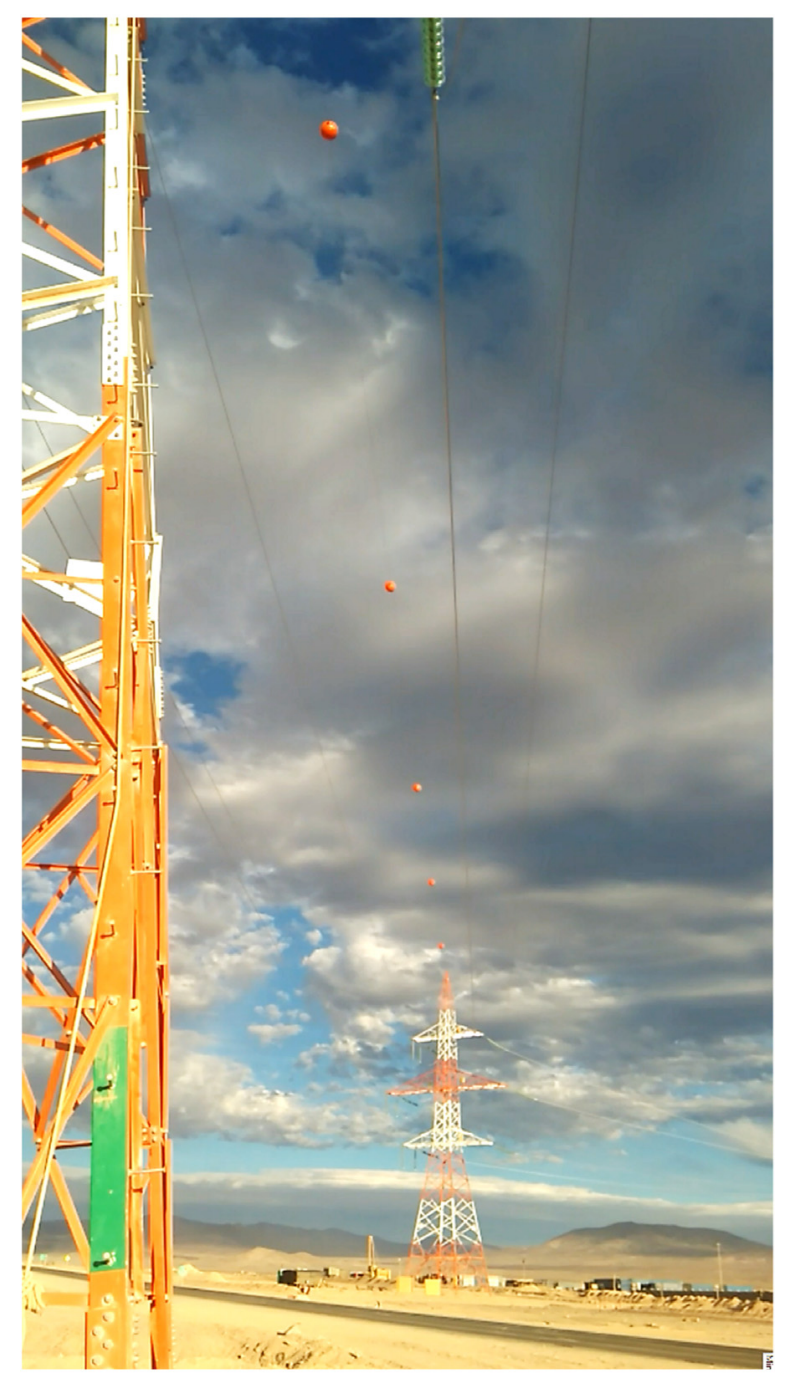

Fig. 1. General view of a guard cable with 5 beacons. Case-1.

The mechanical system considered is sketched in Fig. 5. A cable under tension $T_{0}$ is fitted with $N_{R}$ beacons, which are modeled as points with mass $m$, placed along the cable at $l_{R}$ distance of each other.

In the model, the mass of the cable is incorporated into the beacon mass. Therefore, the modes of the cable itself are not considered, cable segments between beacons are modeled as straight lines. In the field, the displacement amplitudes are in the order of $0.3 \mathrm{~m}$, which compared to beacon separations of $30 \mathrm{~m}$, leads to a relative displacement of some $1 \%$. Therefore, only small amplitude displacements are considered.

Lagrange's equations have been used to study the small amplitude motions of the system. In order to model the effect of the tension, the variation of the potential energy has been considered as the work of the tension when a point mass is displaced an amount $\mathrm{d} q_{1}$. At each end of the cable (Fig. 5a) the contribution to the potential energy of each sub-span can be written as

$$
\mathrm{d} U_{i}=T_{0} \frac{q_{i}}{l_{R}} \mathrm{~d} q_{i} ; \quad U_{i}=\frac{1}{2} \frac{T_{0}}{l_{R}} q_{i}^{2}=\frac{1}{2} k q_{i}^{2} ; \quad k=\frac{T_{0}}{l_{R}}
$$

and at an intermediate sub-span

$$
\mathrm{d} U_{i}=T_{0} \frac{q_{i}-q_{i+1}}{l_{R}} \mathrm{~d}\left(q_{i}-q_{i+1}\right) ; \quad U_{i}=\frac{1}{2} k\left(q_{i}-q_{i+1}\right)^{2}
$$




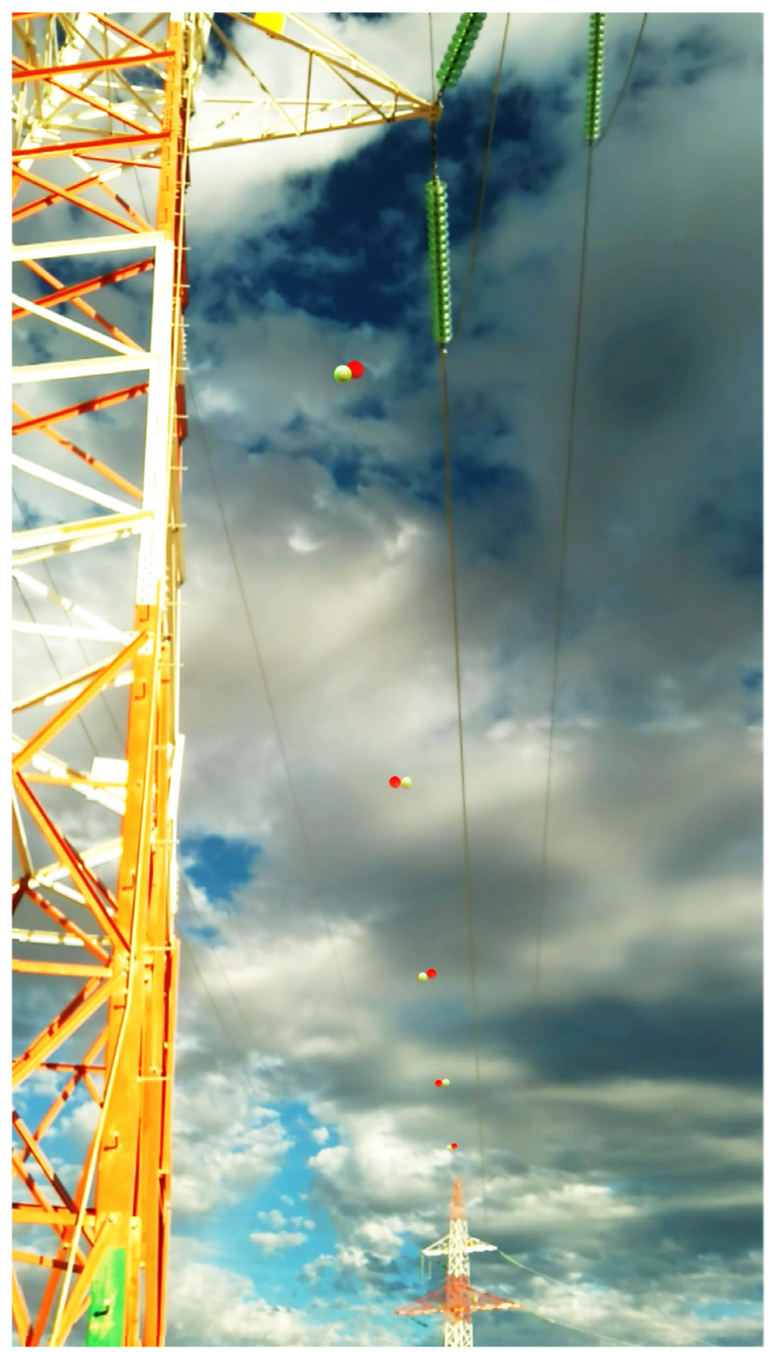

Fig. 2. Close-up view of the guard cable with 5 beacons, obtained from two superimposed frames showing two opposite positions along a whirling turn, with colored beacons: white, frame 1 ; red, frame 2 . The time interval between these two frames is $0.334 \mathrm{~s}$. Case- 1 . Observe the alternative displacement of the consecutive beacons. (For interpretation of the references to colour in this figure legend, the reader is referred to the web version of this article.)
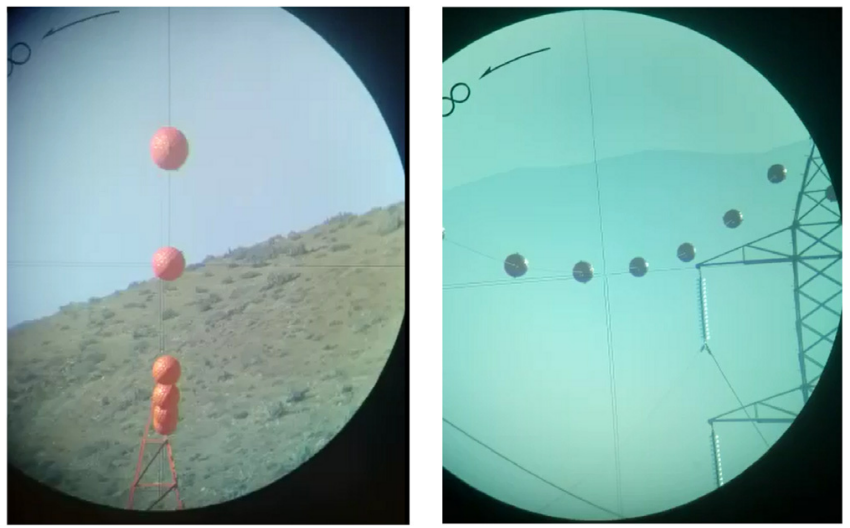

Fig. 3a. Close-up view of a segment of a guard cable with 15 beacons. Left video WA0024, mode A (only 5 beacons are seen); right: video WA0026, mode $\mathrm{B}$ (only 6 beacons are seen). Case-2. Data shown in Table 1 . 
Mode A

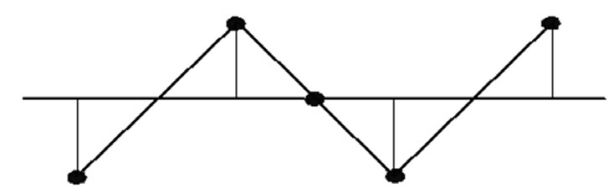

Mode B

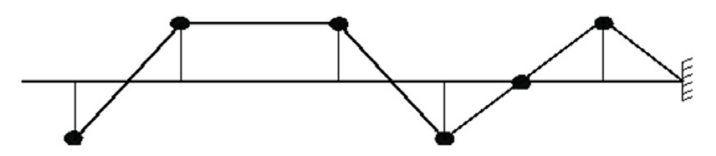

Fig. 3b. Vertical oscillation motion. Mode shapes. Up: 5 beacons of Mode A (WA0024); down: 6 beacons of mode B (WA0026). Case-2. Data in Table 1.

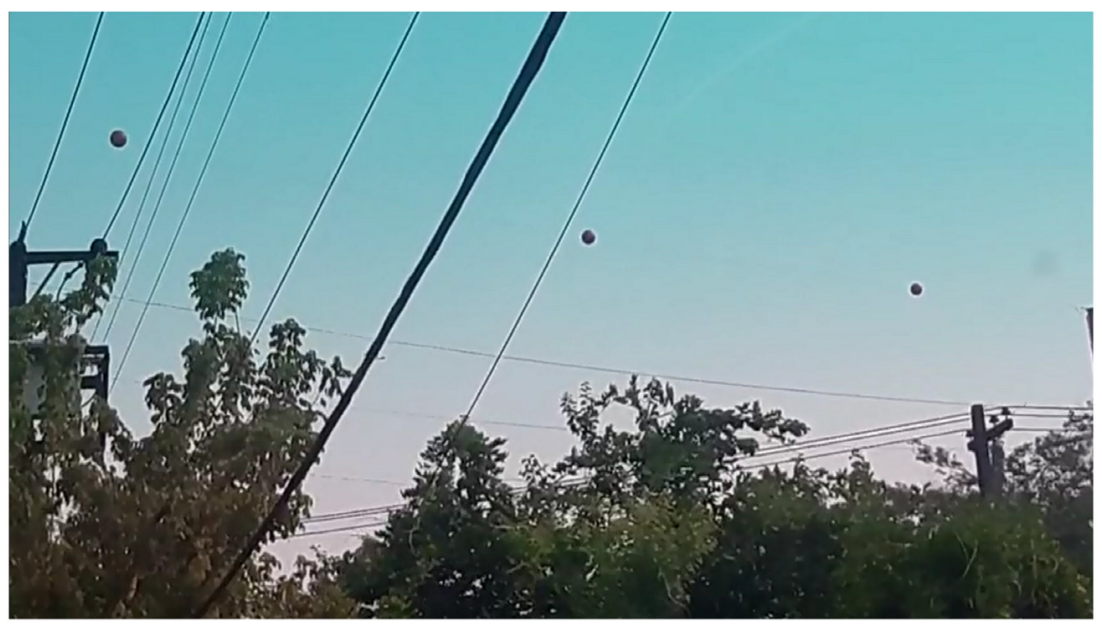

Fig. 4. Close-up view of the guard cable with beacons. WA0007-2017. Case-3.

In the case of $N_{R}$ masses, the kinetic and potential energies are given respectively by

$$
\begin{aligned}
& T=\frac{1}{2} m \sum_{1}^{N_{R}} \dot{q}_{i}^{2} \\
& U=\frac{1}{2} k\left[q_{1}^{2}+\sum_{1}^{N_{R}-1}\left(q_{i}-q_{i+1}\right)^{2}+q_{N_{R}}^{2}\right]
\end{aligned}
$$

Using the Lagrange equation formulation, the following system is obtained

$$
\begin{aligned}
& m \ddot{q}_{1}+k\left[q_{1}+\left(q_{1}-q_{2}\right)\right]=0 \\
& m \ddot{q}_{2}+k\left[-\left(q_{1}-q_{2}\right)+q_{2}\right]=0 \\
& \ldots \ldots \ldots \ldots \ldots \ldots \ldots \ldots \ldots \ldots \ldots \ldots \ldots \ldots \ldots \ldots \ldots \\
& m \ddot{q}_{N_{R}}+k\left[-\left(q_{N_{R}-1}-q_{N_{R}}\right)+q_{N_{R}}\right]=0
\end{aligned}
$$

$$
q_{i}=q_{0 i} \mathrm{e}^{\mathrm{i} \omega t}
$$

by substituting in (3.5), leading to,

$$
\left(-\omega^{2}\left[\begin{array}{cccc}
1 & 0 & \cdot & 0 \\
0 & 1 & \cdot & 0 \\
\cdot & \cdot & \cdot & \cdot \\
\cdot & \cdot & \cdot & \cdot \\
0 & 0 & \cdot & 1
\end{array}\right]+\omega_{0}^{2}\left[\begin{array}{cccc}
2 & -1 & \cdot & 0 \\
-1 & 2 & \cdot & 0 \\
\cdot & \cdot & \cdot & \cdot \\
\cdot & \cdot & 2 & -1 \\
0 & 0 & -1 & 2
\end{array}\right]\right)\left\{\begin{array}{c}
q_{01} \\
q_{02} \\
\cdot \\
\cdot \\
q_{0 N_{R}}
\end{array}\right\}=\left\{\begin{array}{l}
0 \\
0 \\
\cdot \\
0
\end{array}\right\}
$$


a)

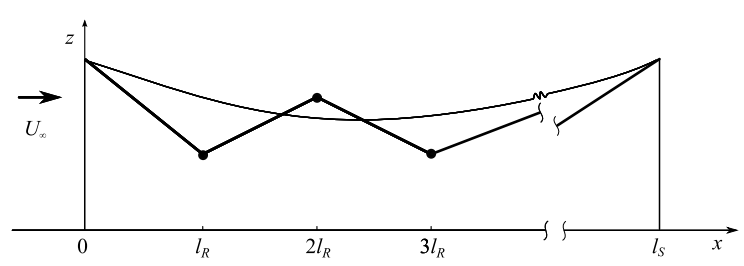

b)

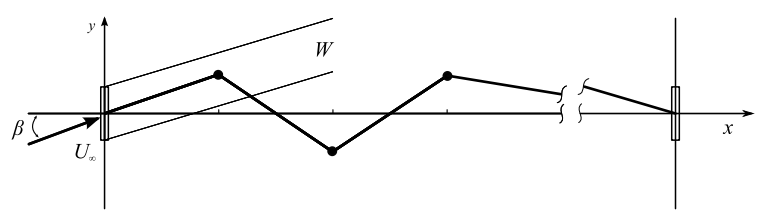

c)
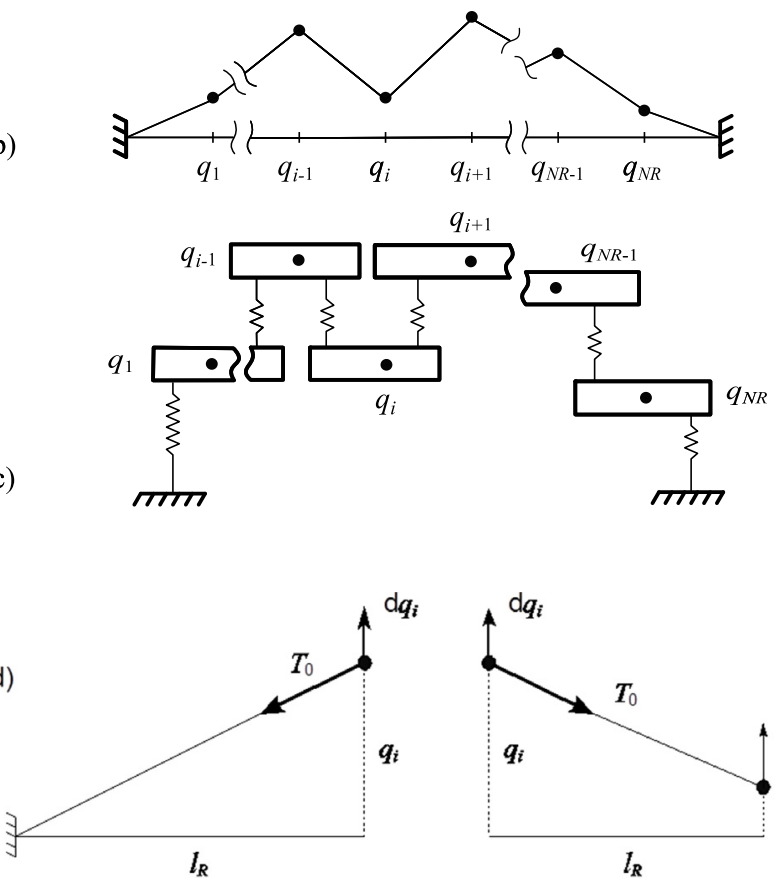

Fig. 5. (a) Sketch of the system considered; (b) idealized system; (c) equivalent system; (d) contribution of each sub-span to the potential energy (effect of the cable tension); left: end sub-span; right: intermediate sub-span. $W$ : wind direction. Cable-wind angle $\beta \ll 1$.

in matrix form. Using new variables $X=\omega^{2} / \omega_{0}^{2}$ and $\omega_{0}^{2}=k / m$, Eq. (3.7) can be written as

$$
\left[D_{N_{R}}\right]\left\{\begin{array}{c}
q_{01} \\
q_{02} \\
\cdot \\
q_{0 N_{R}}
\end{array}\right\}=\left\{\begin{array}{l}
0 \\
0 \\
\cdot \\
0
\end{array}\right\}
$$

where

$$
\left[D_{N_{R}}\right]=\left[\begin{array}{cccc}
2-X & -1 & \cdot & 0 \\
-1 & 2-X & \cdot & 0 \\
\cdot & \cdot & \cdot & \cdot \\
\cdot & \cdot & 2-X & -1 \\
0 & \cdot & -1 & 2-X
\end{array}\right]
$$

This is a block tridiagonal matrix, the main diagonal elements are all equal to $(2-X)$, and the other two contiguous diagonals are -1 .

The eigenvalues $X_{i}\left(i=1 \ldots N_{R}\right)$ are the solutions of $\left|D_{N_{R}}\right|=0$, and the eigenfrequencies are

$$
\omega_{i} / \omega_{0}=\sqrt{X_{i}}
$$


Table 2

Eigenvalues $X=\left(\omega / \omega_{0}\right)^{2}$ and eigenvectors with unitary norm of a guard cable with $N_{R}=5$ beacons, [V]: transposed modal matrix (the eigenvectors are the rows).

\begin{tabular}{|c|c|c|c|c|c|}
\hline$[V]$ & & & & & $\left(\omega / \omega_{0}\right)^{2}$ \\
\hline 0.2887 & 0.5000 & 0.5774 & 0.5000 & 0.2887 & 0.2679 \\
\hline 0.5000 & 0.5000 & 0.0000 & -0.5000 & -0.5000 & 1.0000 \\
\hline 0.5774 & 0.0000 & -0.5774 & 0.0000 & 0.5774 & 2.0000 \\
\hline 0.5000 & -0.5000 & 0.0000 & 0.5000 & -0.5000 & 3.0000 \\
\hline 0.2887 & -0.5000 & 0.5774 & -0.5000 & 0.2887 & 3.7321 \\
\hline
\end{tabular}

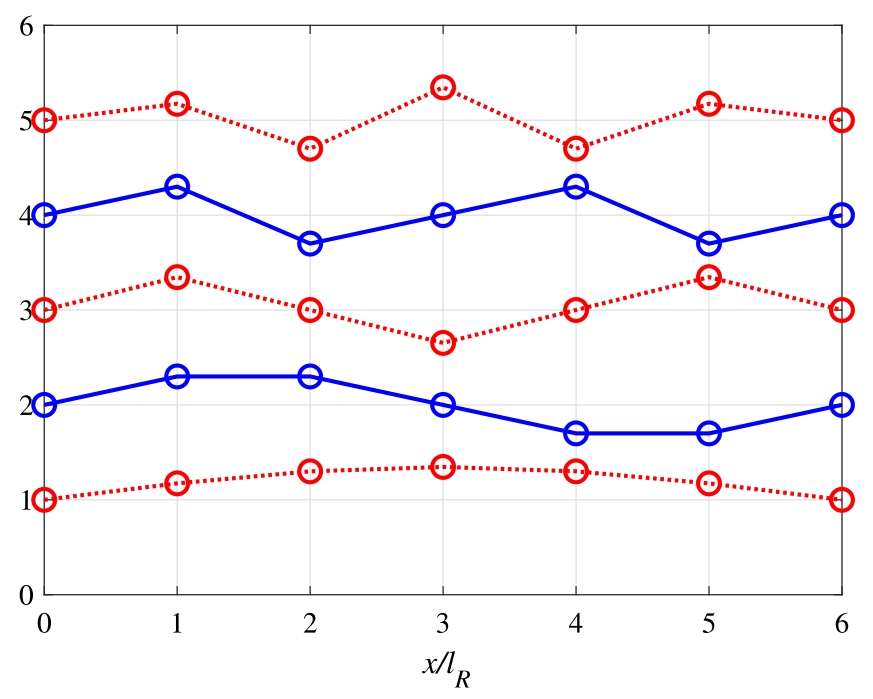

Fig. 6. Eigenvectors (modal shapes) of the deformation of the system with $N_{R}=5$ beacons Circles indicate the displacement of the beacons corresponding to a given mode $N_{M}=1$ to $N_{R}$. The extreme positions, $x / l_{R}=0$ and $x / l_{R}=6$, are the nodes corresponding to the supporting points. $x$ : longitudinal position, $l_{R}$ beacon separation distance. Each mode is plotted at incremental vertical positions.

The eigenvalues and eigenvectors of a configuration with 5 beacons are presented in Table 2, and sketched in Fig. 6, so that the mode shape of this oscillation mode can be compared with the mode shape of the whirling motion shown in Fig. 2 (see Section 2, Case 1). The results for a configuration of 15 beacons (Case-2) are presented in Figs. 7 and 8.

As the number of beacons $N_{R}$ increases, the number of modes of each configuration also increases (the maximum value of the number of modes $N_{M}$ is $N_{R}$ ), and the lower order mode shapes approach more closely the deformation of a continuous beam (as they contain more points in each wavelength). The eigenvalue order number $N_{M}$ coincides with the number of half waves of the deformation. The odd modes, as they contain an odd number of half waves, are symmetrical (with regard to the mid span point) and vice versa.

As shown in Fig. 6, the lowest mode always has all beacons with the same sign displacement, the antinode being in the middle. As shown in Figs. 6 and 7, the highest mode always has adjacent beacons with opposite displacement signs. This mode shape is the one identified in the autorotation cases reported.

The dimensionless frequency of the highest mode (for $N_{M}=N_{R}$, alternative displacements) $\sqrt{X_{N_{R}}}$ tends towards 2 , as the number of beacons $N_{R}$ increases (see Fig. 8). In the limit $N_{R} \gg 1$, the mode shape is very close to a single mass with a cable length $l_{R} / 2$ at each side, repeated $N_{R}$ times, therefore $\omega_{\lim }^{2}=2 \mathrm{k} / \mathrm{m}=4 T_{0} /\left(l_{R} m\right)=4 \omega_{0}^{2}$.

For small amplitude motions the effect of gravity can be ignored if the cable tension is high enough. In the limit of a large cable tension (shallow catenary), there is no difference between vertical and horizontal oscillations. Otherwise, when this limit is not applicable, the influence of gravity should be larger on the horizontal oscillations (pendulum effect); this case will be studied in the future.

Therefore, Eq. (3.5) can be used to describe both horizontal and vertical oscillations, with the same eigenfrequencies. If both motions are excited at the same time, they can appear as combined, leading to a whirling motion in the transverse plane, with the same frequencies as predicted by the model for the oscillatory motions (Table 2 and Fig. 8).

For the beacons to keep whirling, a minimum revolving speed is needed, as the gravity action has to be compensated by the centrifugal acceleration, $\omega_{g}=\sqrt{g / r_{g}} \approx \sqrt{9.8 / 0.3} \mathrm{~s}^{-1}=5.7 \mathrm{~s}^{-1}$, that is $f_{g}=0.9 \mathrm{~Hz}$, where $r_{g}$ is the revolving radius, which in the reported cases is of the order of half a diameter of the beacon, $r_{g}=0.3 \mathrm{~m}$. The existence of this limit could be the reason why in rotation only the higher mode shapes are observed: lower frequency modes cannot appear as whirling modes as they could not overcome the action of gravity. Note that all the reported whirling frequencies (see Section 2) are larger than $0.9 \mathrm{~Hz}$. Interestingly, this whirling motion has only been reported for the highest mode shape $N_{M}=N_{R}$, associated to the highest frequency mode, and the only one that has alternative displacements. 


\begin{tabular}{lllll}
\hline Symbol & Definition & Units & $N_{R}=9$ & $N_{R}=15$ \\
\hline$m_{B}$ & Beacon mass & $\mathrm{kg}$ & 4.7 & 4.7 \\
$\rho_{c}$ & Cable density (mass per unit length) & $\mathrm{kg} / \mathrm{m}$ & 0.6 & 0.6 \\
$l_{S}$ & Span length & $\mathrm{m}$ & 300 & 459 \\
$l_{R}$ & Beacon separation distance & $\mathrm{m}$ & 30 & 30 \\
$T_{0}$ & Tension & $\mathrm{m}$ & 6000 & 15000 \\
$d_{B}$ & Beacon diameter & $\mathrm{mad} / \mathrm{s}$ & 0.6 & 0.6 \\
$\omega_{0}{ }^{a}$ & Reference angular frequency & $\mathrm{Hz}$ & 4.32 & 6.8 \\
$f_{0}$ & Reference circular frequency & - & 0.69 & 1.08 \\
- & Reported modes & Transverse whirling & Vertical oscillation \\
\hline
\end{tabular}

${ }^{\mathrm{a}}$ See Eq. (4.1).

\section{Analysis of reported cases}

The available data for the configurations considered (guard cables with 9 and 15 beacons, respectively) is given in Table 3 . With the guard cable data in Table 3, the reference angular frequencies can be calculated by using

$$
\omega_{0}=\sqrt{\frac{k}{m_{e}}}=\sqrt{\frac{T_{0}}{l_{R} m_{e}}}
$$

where $m_{e}=m_{B}+r_{m} l_{R} \rho_{c}$ is the equivalent mass, and the participation coefficient has been assumed as $r_{m}=1 / 3$. The participation coefficient considered here is the fraction of the cable mass that represents the contribution of this mass to the oscillation motion. It is an analogy of the concept used in the case of vibrations of elastic systems (Meirovitch, 2001) with springs that have non-zero mass (non-ideal springs). When the kinetic energy of the spring is taken into account (assuming that the mass is uniformly distributed), the participation coefficient obtained is $r_{m}=1 / 3$.

The reference circular frequency is given by

$$
f_{0}=\frac{\omega_{0}}{2 \pi}
$$

\subsection{PC case}

In the PC field case, with the number of beacons $N_{R}=9$, the transverse whirling motion shows an instantaneous deformation with 9 half waves (alternative displacement), very similar to the oscillation mode $N_{M}=9$. The largest eigenvalue in this case is $X_{9}=3.9$ and the corresponding dimensionless frequency is $\sqrt{X_{9}}=1.97$. The dimensional frequency for $N_{M}=9$ is given by

$$
f_{9}=\sqrt{X_{9}} f_{0}=1.36 \mathrm{~Hz}
$$

The observed frequency in the video sequences is $f_{9}=1.5 \mathrm{~Hz}$, which is close to the value obtained from Eq. (4.3). The model frequency $f_{9}$ could be better fitted to the actual value, by tuning the cable mass participation coefficient value. Here it has been taken as $r_{m}=1 / 3$, which is an estimated value obtained from the mass-spring elastic model for a non-ideal spring.

\subsection{Case-2}

The data of the guard cable in Case-2 (with $N_{R}=15$ beacons) is given in Table 3 . The 10 highest mode shapes $\left(N_{M}=6\right.$ to 15), obtained with the model presented in Section 3, are shown in Fig. 7, and the eigenfrequencies are plotted in Fig. 8. The results for the two reported modes (A, B in Fig. 3b) are summarized in Table 4. The identification of these modes is not easy because they span only a fraction of the whole mode shape (see Fig. $3 \mathrm{~b}$ ). The only way we realized to achieve this was to compare it with the "catalogue" of theoretical mode shapes plotted in Fig. 7. The observed mode shape segments A, B can be identified as a segment of 12 th mode (beacons between $x / l_{R}=10$ and 14 ) and 9th mode (beacons between $x / l_{R}=10$ and 15), respectively. The identification is possible thanks to that the extension of the observed deformation shape segment is large enough to allow an unambiguous comparison; although incomplete, the segment includes more than one complete wavelength. In the previous discussion we are assuming that the aerodynamic forces do not change the elastic mode shapes. The aerodynamic loads have a local character, so that they follow the motion of each beacon at an individual level, without influencing the elastic modal shape.

The frequency ratio given by the elastic model is $f_{12} / f_{9}=1.19$, and in the actual cable is $f_{e A} / f_{e B}=1.23$, close to each other. The reference frequency can be estimated as $f_{0}=f_{\mathrm{eB}} /\left(f_{9} / f_{0}\right)=0.92 \mathrm{~Hz}$, which is relatively close to the value $f_{0}=1.08$ given in Table 3. It should be considered that the participation coefficient affects the absolute value of the frequency but it has no influence on the frequency ratio $f_{12} / f_{9}$. This difference in the fitting degree suggests that it would be interesting to devote further work to improve the determination of the participation coefficient of these configurations. 
Table 4

Results for the two reported vertical oscillation modes in Case-2 (see Fig. 3). Mode label (see Fig. 3b), mode number $N_{M}$ (see Fig. 7), mathematical model dimensionless frequency $f \mid f_{0}$ (Table 2 ), field data reported frequency $f_{e}$.

\begin{tabular}{llll}
\hline Mode label & $N_{M}$ & $f / f_{0}$ & $f_{e}[\mathrm{~Hz}]$ \\
\hline $\mathrm{A}$ & 12 & 1.85 & 1.76 \\
$\mathrm{~B}$ & 9 & 1.55 & 1.43 \\
\hline
\end{tabular}

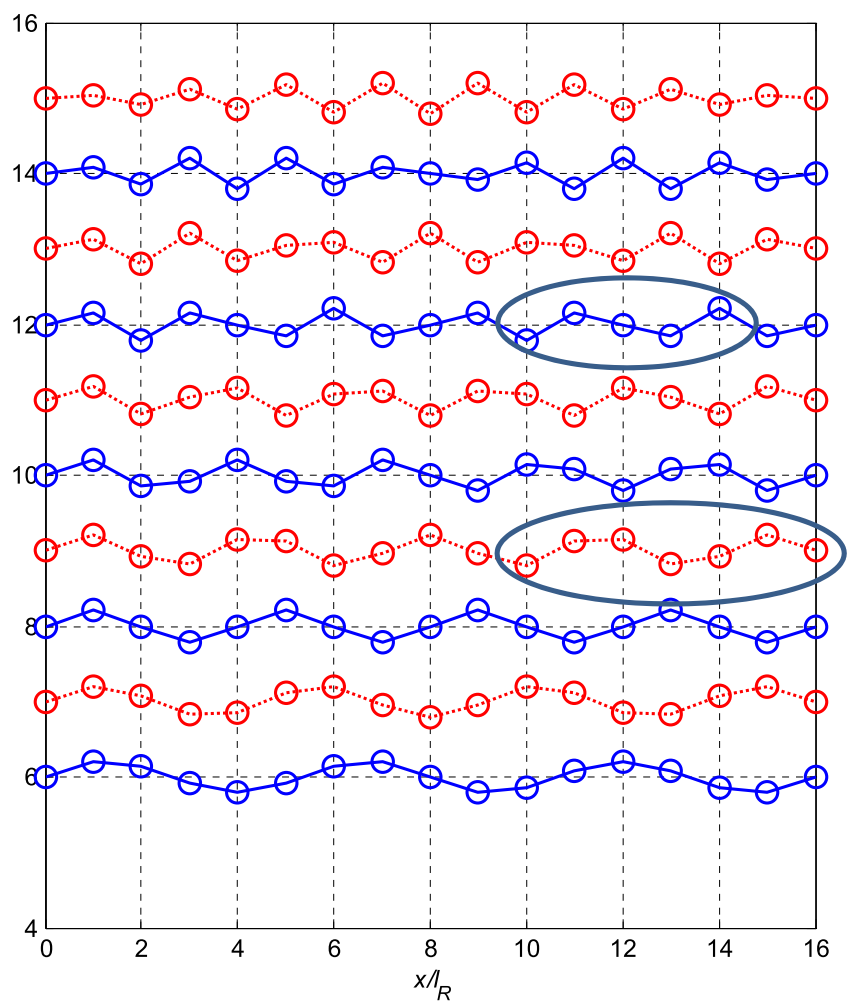

Fig. 7. As Fig. 6 for a system with $N_{R}=15$ beacons (Case-2). Only the 10 highest eigenmodes (from $N_{M}=6$ to 15 ) are plotted, for convenience. Modes A and B of Fig. 3b are outlined. Results from the mathematical model.

\section{Aerodynamic loading}

The elastic system moves in response to the action of aerodynamic loading. As in the reported motions the beacons had a spherical shape, galloping was not considered as a good candidate for the aerodynamic load, although the supporting cable could introduce some kind of asymmetry that could explain a variation of aerodynamic load with the angle of attack. Thus, the vortex induced vibration instability (VIV) was considered as the most suitable phenomenon to explain the oscillation mode of TILoW instability in the subcritical Reynolds number Re range. Force excitation frequency due to VIV action can be estimated using the Strouhal number $\mathrm{St}=f_{v o} d_{B} / U_{\infty}$ where $f_{v o}$ is the frequency of vortex shedding, $d_{B}$ the beacon diameter, and $U_{\infty}$ the incoming flow speed.

The Strouhal number for a static sphere is $\mathrm{St}=0.18 \pm 0.01$ (Achenbach, 1974), in the Reynolds number range considered $4 \cdot 10^{4}<\operatorname{Re}<2 \cdot 10^{5}$, which corresponds to wind speeds $1 \mathrm{~m} / \mathrm{s}<U_{\infty}<5 \mathrm{~m} / \mathrm{s}$. For the cases reported, the reference frequency of oscillation $f_{0}$ is in the range from $0.7 \mathrm{~Hz}$ (for $N_{R}=9$ ) to $1.1 \mathrm{~Hz}$ (for $N_{R}=15$ ), as shown in Table 3. The corresponding reference wind speed $U_{\infty 0}$ needed to excite these vortex shedding frequencies is close to $3 \mathrm{~m} / \mathrm{s}$. The eigenfrequencies of the elastic system (Fig. 8) are in the range between $0.2 f_{0}=0.18 \mathrm{~Hz}$ and $2 f_{0}=1.8 \mathrm{~Hz}$, which is variation with a large dynamic range $(1.8 \mathrm{~Hz} / 0.18 \mathrm{~Hz})$. Accordingly, the range in wind speed $U_{\infty}$ for synchronization spans from $0.6 \mathrm{~m} / \mathrm{s}$ to $6.0 \mathrm{~m} / \mathrm{s}$, which is a range of wind speed values with a high probability of occurrence.

It could be conjectured that the two modes in Case-2, A and B, respectively (see Table 4), could have been excited by longitudinal winds with different speeds, $U_{\mathrm{A}}$ and $U_{\mathrm{B}}$, such as $U_{\mathrm{A}} / U_{\mathrm{B}}=f_{12} / f_{9}=1.19$.

From a general point of view, on one side, the effect of increasing the beacon number is to increase the number of degrees of freedom of the mechanical system, and therefore, the number of eigenfrequencies (and eigenmodes) that can be excited, see Fig. 8. On the other side, wind can produce some aerodynamic loading in the beacons (like VIV) at a given frequency (if the 


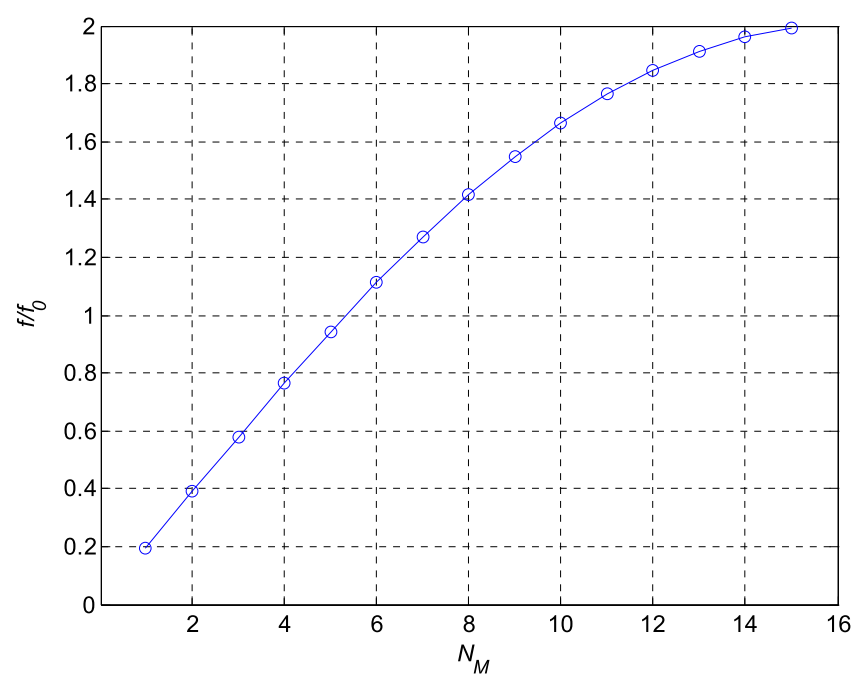

Fig. 8. Dimensionless eigenfrequencies $f / f_{0}$ of an elastic system with $N_{R}=15$ beacons, as a function of mode number $N_{M}$. Results from the mathematical model.

beacons all have the same diameter). Therefore, the number of specific wind speed values that can synchronize the elastic system motion to the VIV excitation (through one of the available elastic resonance frequencies of the system) increases in proportion to the number of beacons. Note the small interval between resonant frequencies in Fig. 8. To further complicate the problem, the amplitude of the synchronization range, or "lock-in", around each resonant wind speed helps to increase the range of instability persistence.

One could expect other types of motions. For instance, vortex shedding from a sphere is expected to be omnidirectional because of the obvious 3-D symmetry, and this could have excited other modes. However, the omnidirectionality is true for a static sphere, but for a moving sphere (or with moving elements) it seems (see Section 6) that there is a preference for the orientation of the wake (Norman et al., 2011a,b). In the case of beacons, the cable also introduces a breaking of this 3-D symmetry (Provansal et al., 2004).

In-line oscillations could also have been expected, but the motion is much more restricted in the longitudinal direction than in the transverse direction, because of the differences of stiffness in each direction. The longitudinal stiffness is a result of the large mechanical tension applied to the guard cable.

The explanation of the collective autorotation mode needs further research work, which is under way.

\section{Experimental test with a simplified configuration}

In order to clarify the problem of a guard cable with multiple beacons, we will consider the simplified case of a cable with a single beacon, because the kinematic and elastic descriptions are far simpler (just a pendulum) while the aerodynamic aspects are quite similar.

The single beacon problem can be described as an oscillating swinging sphere (SS), longitudinally blown. Looking for a reference, the closest aerodynamic problem that has been found in the literature is the tethered sphere (TS). In both cases, SS and TS, we have to deal with the flow generated by a uniform stream around a sphere that is performing transverse oscillations around a given central position. Cinematically, the single swinging sphere motion is a single pendulum, driven by gravity and restrained to the plane perpendicular to the swing rotation axis; on its side, the tethered sphere is a spherical pendulum, although for heavy spheres, the motion of the tether seems to be restrained to a plane, at a given angle with the mainstream.

The behavior of a tethered sphere (of smooth surface) has been reviewed and explained by Govardhan and Williamson (2005), using the reduced wind speed $U^{*}=U_{\infty} /\left(f_{R} d_{B}\right)$ and the reduced mass $m^{*}=m / m_{d}$. For smooth spheres it seems that there is not much influence of Re in the range $2.000<\operatorname{Re}<30.000$. Three oscillation modes were found: Mode I $\left(4<U^{*}<6\right)$, Mode II $\left(6<U^{*}<10\right)$ and Mode III $\left(12<U^{*}\right)$. Modes I and II correspond to oscillations at the pendulum natural frequency, $f_{R}$. At low flow speed there is no oscillation motion until $U^{*}=1 / \mathrm{St}$ is reached, where oscillation starts and synchronization between vortex shedding frequency and fundamental pendulum's frequency is obtained $\left(U^{*} \cong 4\right.$, depending on Re). As fluid velocity increases, the oscillation frequency remains the same $f=f_{R}$, and the amplitude increases, until a maximum is reached at $U^{*} \cong 8$. There is no transition between Mode I and Mode II for $m^{*}$ large, but a clear transition appears at $U^{*} \cong 6$ for small values of $m^{*}$. For large $m^{*} \cong 100$ the synchronization at constant frequency $f=f_{R}$ is presented, but for small values of $m^{*} \cong \mathrm{O}(10)$, the synchronization frequency $f$ changes almost linearly as a function of $U^{*}$. The phenomena that appear for low $m^{*}$ are very interesting, but they are outside the scope of the present work. 
Concerning the wake, these authors pointed out that, in the case of a static sphere, the wake structure is wandering behind the spheres, while in the induced vibration of a sphere the vortex loops lock in a particular preferred azimuthal orientation, which satisfy the condition of symmetry with regard to the plane defined by the sphere center displacement line and the flow direction.

In some cases, the sphere and cylinder VIV dynamics are compared, but they are very different. The cylinder performs oscillations only at the fundamental synchronization frequency $4<U^{*}<12$ while the sphere can oscillate (with large amplitude and increasing frequency) also at large reduced velocity $U^{*}$ (Mode III).

In order to obtain data on the dynamics of the SS simplified configuration, experimental tests were performed in a wind tunnel as described in the following subsection. We will try to compare the results obtained here for SS with the results of Govardhan and Williamson (2005) for TS as much as possible.

\subsection{Experimental setup and procedures}

Tests were conducted at the ACLA16 wind tunnel of IDR/UPM. This is a low-speed closed return wind tunnel with a closed test chamber, which has a squared cross section, $2.2 \mathrm{~m}$ side and $20 \mathrm{~m}$ length (Fig. 9). Sixteen fans drive the wind tunnel, 7.5 $\mathrm{kW}$ each one (the total power is $120 \mathrm{~kW}$ ). The wind speed is controlled electronically by means of a variable frequency drive, and flow velocities up to $32 \mathrm{~m} / \mathrm{s}$ can be attained.

A schematic of the experimental set-up is shown in Fig. 10. A cable that simulates the catenary has been fixed between two supports separated by a distance $l_{S}$ and aligned with the direction of the flow generated by the wind tunnel. The support located upstream consisted of two very thin steel cables tensioned from each corner of the test chamber $\left(45^{\circ}\right.$ inclined cross) so that they intersected at the center of the test section. This support minimizes aerodynamic interference on the model. Downstream, the catenary has been fixed to a linear translation stage, which allows the sag of the catenary, $r_{g}$, to be adjusted. The beacon model was placed in the test chamber center, where the flow is practically uniform.

Three displacement laser sensors manufactured by MEL (model M7L/100) were pointed directly at the beacon: two were placed to point at each one of the sphere transverse sides, so that they could measure the lateral displacements, and the third one was placed below pointing upwards so that it could measure the vertical displacement. At each wind velocity, measurements were taken at $1 \mathrm{kHz}$ sampling rate, during $60 \mathrm{~s}$. The amplitude of the motion was determined based on the root mean square (rms) of the displacement record, once the zero values (measured at rest conditions) were subtracted. No additional filtering was performed. The power spectral density (PSD) of the displacements at each wind speed were computed using FFT algorithm. The whole $60 \mathrm{~s}$ register was used. No additional processing of the spectrum was performed.

Pictures of the set-up are shown in Fig. 11.

In addition, the incident flow speed has been measured by using a hot wire probe model 55p 16 from Dantec Dynamics located upstream. The measurement system was completed with an acquisition card, a Dantec Dynamics CTA 90C10 module, and a computer. All the velocity measurements were carried out at a rate of $1 \mathrm{kHz}$ during a sampling time of $60 \mathrm{~s}$.

The hot wire sensor was placed $4 \mathrm{~m}$ upstream the test model, at some $0.4 \mathrm{~m}$ above the tunnel floor.

From the data obtained of hot-wire measurements the approaching flow has been characterized. Flow speed was approximately uniform with a longitudinal turbulence intensity $I_{u}=\sigma_{u} / U_{\infty} \approx 1.8 \%$, where $\sigma_{u}$ is the standard deviation of the wind velocity fluctuation time series and $U_{\infty}$ is its mean value. The reduced power spectral density (PSD) of turbulent fluctuations, $u$, as a function of reduced frequency is shown in Fig. 12, for a test in which the average velocity was $U_{\infty}=9.4$ $\mathrm{m} / \mathrm{s}$. In this figure $S_{u}(n)$ is the PSD, $n$ the frequency, and $L_{u}$ the integral length of longitudinal turbulence. The von Karman spectrum model has been included in the same figure, which can be expressed as:

$$
\frac{n S_{u}(n)}{\sigma_{u}^{2}}=\frac{4 n L_{u} / U_{\infty}}{\left[1+70.8\left(n L_{u} / U_{\infty}\right)^{2}\right]^{5 / 6}}
$$

This model, based on the theory of isotropic turbulence, is widely used in wind engineering applications for power spectra description (American Society of Civil Engineers, 2012; von Kármán, 1948). The integral length of longitudinal turbulence fluctuations has been obtained by fitting the von Karman model to the measured spectra; the value $L_{u} \approx 0.09 \mathrm{~m}$ is obtained, a constant for the whole range of wind velocities covered in the test.

The spheres used were made of polystyrene, with a diameter $d_{B}=0.115 \mathrm{~m}$, sphere mass $m_{B}=70 \mathrm{~g}$, and $m^{*}=89$, to match the field conditions. The configuration selected is defined by the revolving radius $r_{g}=0.08 \mathrm{~m}$ and the span length $l_{S}=1 \mathrm{~m}$, because it helps to show a wide range of motion behaviors. Other parameters are $f_{R}=\omega_{\mathrm{g}} /(2 \pi)$, cable density $\rho_{\mathrm{c}}=1 \mathrm{~g} / \mathrm{m}$, cable tension $T_{0}=8.8 \mathrm{~N}$.

\subsection{Motion of a swinging sphere}

The signals obtained by the displacement sensors during a typical test $y(t)$ are shown in Fig. 13 , together with the respective spectral density $S_{y}$. The variation of the dimensionless amplitude $A_{y}^{*}=\sqrt{2} y_{r m s} / d_{B}$ and the dimensionless frequency $f^{*}=f / f_{R}$ as a function of the reduced speed $U^{*}$ are shown in Fig. 14 . With this definition, in the case of a sinusoidal vibration, dimensionless amplitude $A_{y}^{*}$ coincides with the wave amplitude.

The shape of the signal $y(t)$ and of its power spectrum allow us to distinguish between different types of motion that appear as the flow velocity increases. A total of 7 different types of behaviors (or patterns) have been identified, and are presented in Table 5. The experimental points are labeled with a number in Fig. 14. 


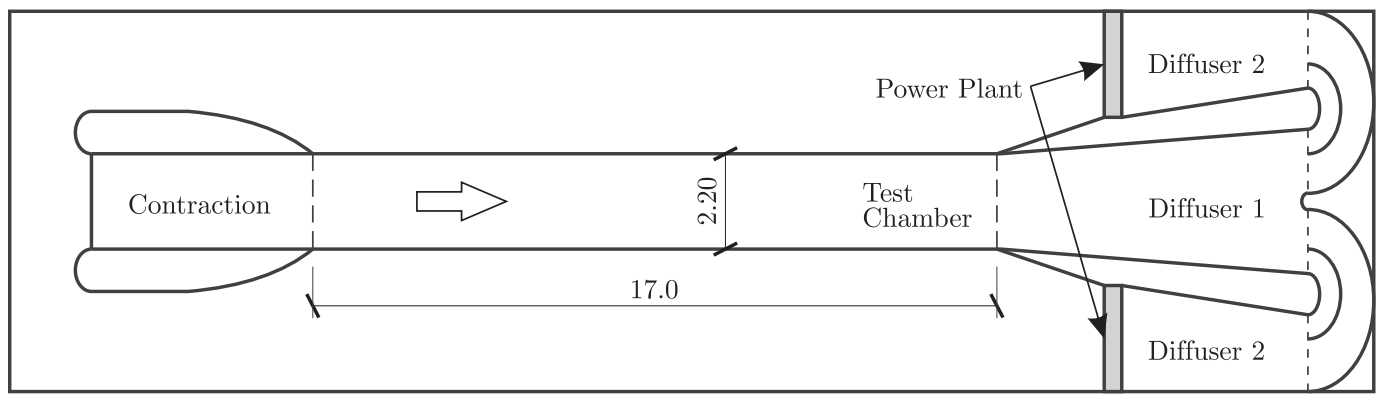

Plant view

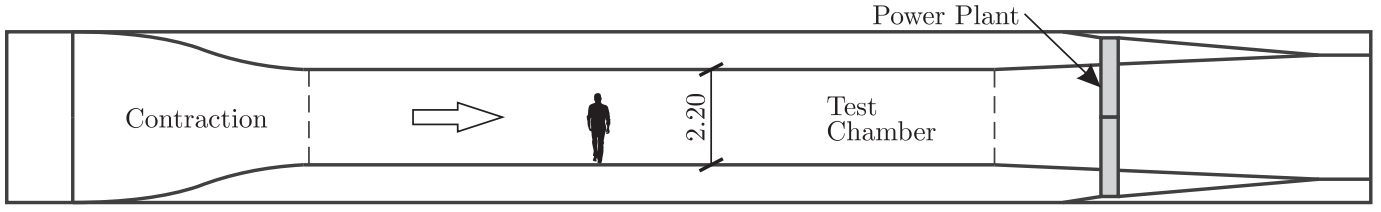

Side view

Fig. 9. Sketch of IDR ACLA-16 wind tunnel.

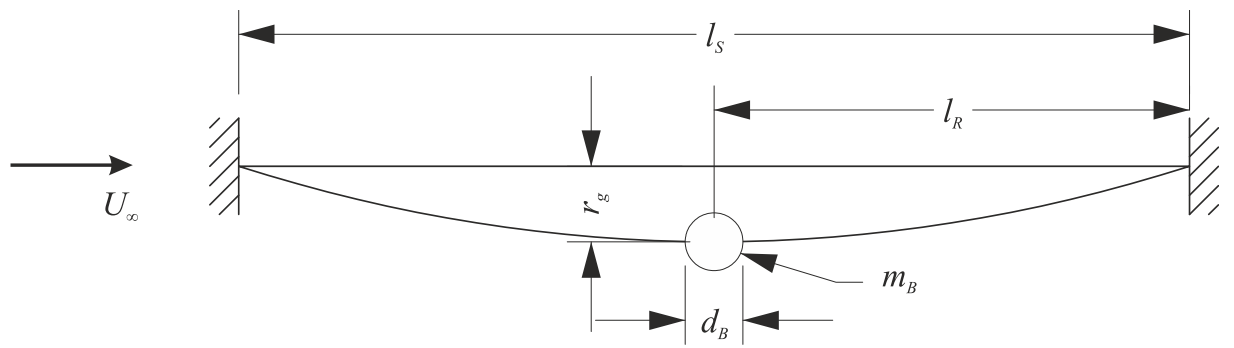

Fig. 10. Sketch of the experimental set-up. Definition of geometric parameters.
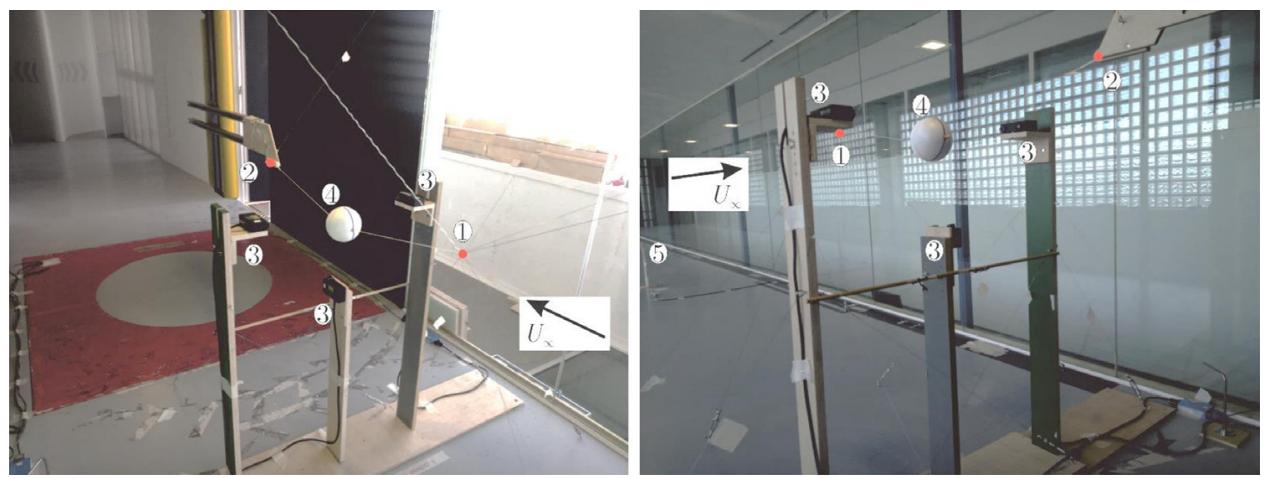

Fig. 11. Experimental setup installed in the IDR ACLA-16 wind tunnel. (1) Upstream support, (2) downstream support, (3) laser distance sensors, (4) swinging sphere and (5) hot wire probe.

The first pattern (0-A) appears at low speed, $U^{*}<1 / \mathrm{St}$, and is characterized by the absence of motion.

In the second pattern (A-B) or "Synchronized oscillation" as a result of Vortex Induced Vibration (VIV) the oscillation motion is quite regular and the amplitude is almost a constant (Fig. 13 \# 3 ). In the power spectrum a peak at $f=f_{R}$ is clearly seen (together with its harmonics). The amplitude increases from $A$ as $U^{*}$ increases, the maximum amplitude is obtained for $U^{*}=13$ (point \# 3) and then the amplitude decreases again until point B is reached. In this process the amplitude starts to fluctuate with time (see point \# 5). The maximum amplitude is $A_{y}^{*}=0.3$. This mode, spanning the $U^{*}$ range $4-27$, is similar to the first modes (Mode I and Mode II) for TS described by Govardhan and Williamson (2005), which appears in the range 4-10. Therefore, the upper limit for SS, $U^{*}=27$, is considerably larger than for TS, $U^{*}=10$, which suggests that the locking capacity of VIV in SS configuration is more powerful than in TS configuration. 


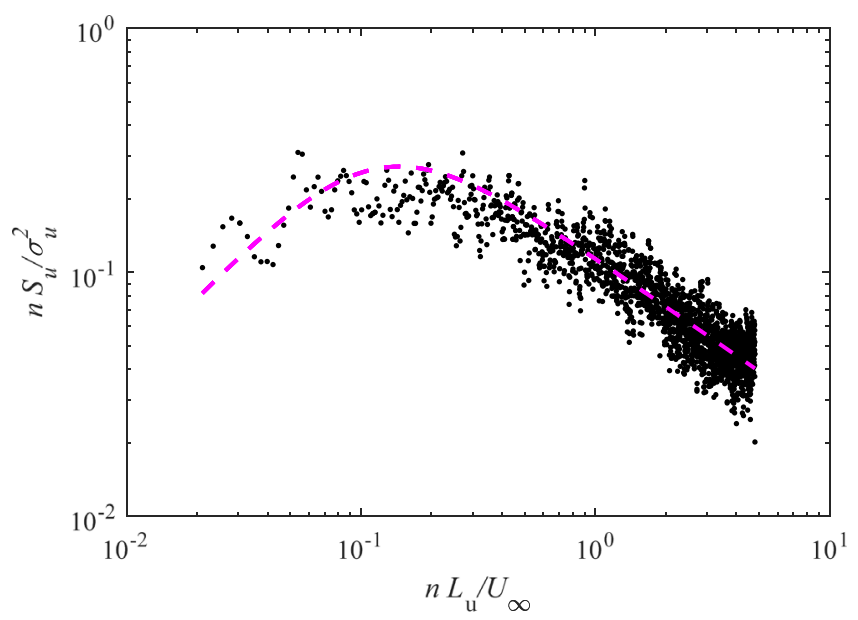

Fig. 12. Reduced power spectral density of turbulent fluctuations as a function of reduced frequency; Von Karman's model (dashed line).

Table 5

Swinging sphere observed response behaviors..

\begin{tabular}{lllll}
\hline Speed range & Response behavior & Comment & Points (Fig. 14) & $U^{*}$ \\
\hline 0-A & 1 Unexcited & No motion. & 1 & $0-4$ \\
A-B & 2 Synchronized Oscillation & Synchronized oscillation by VIV, almost constant amplitude. & $2-4$ & $4-27$ \\
B-C & 3 Transition T1 & As previous, with deep beating. & $5-8$ & 9 \\
C-D & 4 Transition T2 & Intermittency between central and off-center oscillations. & $27-52$ \\
D-E & 5 Transition T3 & Intermittency between off-center oscillation and autorotation. & $10-11$ & $12-17$ \\
E-F & 6 Autorotation & Whirling motion in the transverse plane. & $56-64$ \\
F $<$ & 7 Off-center oscillation & The lateral offset of the oscillation center is some 3 cm. & $18-20$ \\
\hline
\end{tabular}

In the third pattern "Transition T1" (B-C), the oscillation frequency is maintained, $f=f_{R}$, the amplitude strongly fluctuates, and the shape of $y(t)$ can be described as a constant frequency carrier modulated by a deep beating. In the power spectrum this phenomena is translated as a large decrease of the peak intensity at $f=f_{R}$ (by a factor of $1 / 30$ in \#5), and significant growth (by a factor of 10) of the part of spectrum at low frequency $f<f_{R}$. The motion is no longer a single harmonic and the amplitude should be defined in terms of rms. The rms amplitude increases almost linearly with $U^{*}$. The distortion of the signal increases as $U^{*}$ increases until \#8 is reached (the point at the end of this mode). Although the system is oscillating at the resonant frequency, VIV oscillations are no longer a stable motion, and the system tries to jump to another more stable motion.

As the fourth pattern "Transition T2" (C-D) is reached the mean position of the oscillation fluctuates intermittently between two points (Fig. 13 \#9), one is the center of the normal oscillations, $y=0$, and the other is an off-center position, $y=-0.02 \mathrm{~m}$. In the power spectrum the peak at $f=f_{R}$ has almost disappeared and a striving peak appears at low frequency $(f \simeq 0.13 \mathrm{~Hz}$ ) which corresponds to the frequency of the intermittence cycle.

The fifth pattern "Transition T3" (D-E) is also an intermittency but in this case (Fig. 13 \# 10) between off-center oscillation and autorotation. In whirling autorotation the amplitude of the motion is large, $\left|y_{\max }\right|=r_{g}$, the laser sensor becomes saturated and the output signal is cut when the measurement range limits are reached. Therefore, the FFT of this signal is not accurate, but is satisfactory for the purpose. The system keeps trying to jump to another more stable motion.

The sixth pattern "Autorotation" (E-F) is characterized by the whirling motion (in the transverse plane) of the sphere center around the support line, obviously with constant amplitude $r_{g}$, the revolving radius. As the flow velocity is increased this autorotation mode is lost, and passes to the seventh pattern, an off-center oscillation (at the pendulum frequency $f=f_{R}$ ) with an irregular amplitude.

\section{Conclusions}

A new transverse aeroelastic instability that appears in guard cables with beacons when excited by a longitudinal wind (TILoW for short) has been recently reported to the authors. Trying to clarify the problem, in this paper, the aeroelastic instability of the configuration is analyzed, based on the study of the small amplitude oscillation motion of the elastic configuration, the eigenmodes and eigenvalues of the deformation are determined, which helps to explain the coupling with the aerodynamic forces.

The information on this instability has been obtained from a set of videos taken by casual observers. Vertical and horizontal oscillations, and transverse whirling autorotation have been analyzed. It is worth noting that the combination 


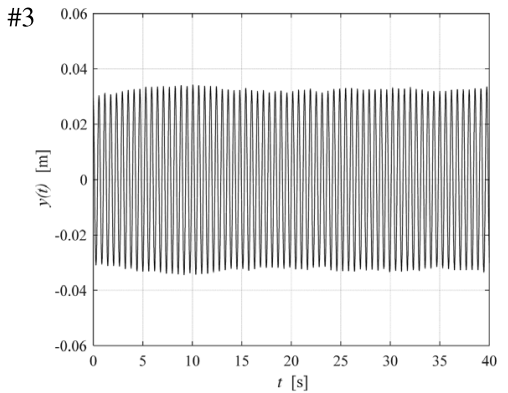

\#5

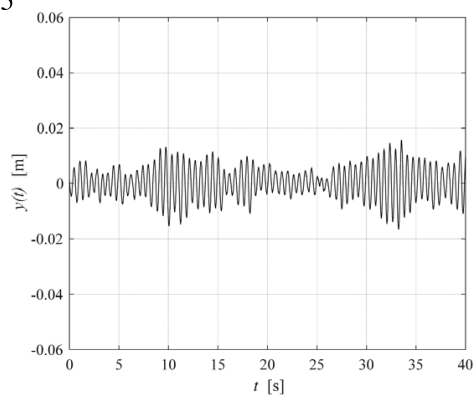

\#8

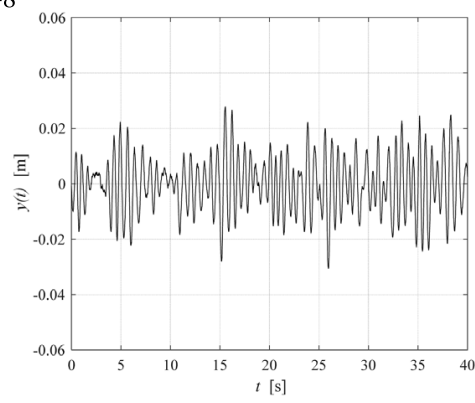

\#9
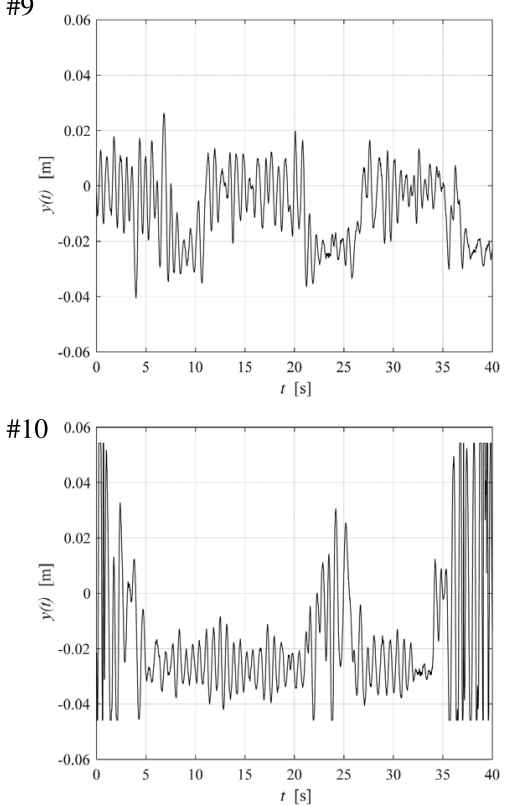
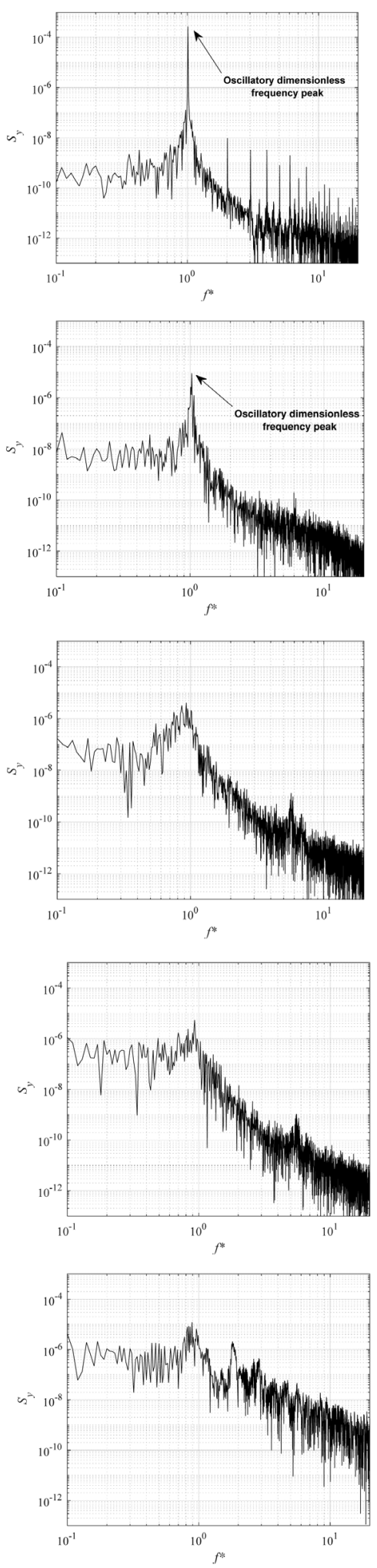

Fig. 13. Left: variation of the dimensionless lateral position $y$ measured with the laser displacement sensors, as a function of time, $t$. Right: Spectral density $\left[\mathrm{m}^{2} / \mathrm{Hz}\right]$ of the position $y(t)$. Points \#3 to \#10 correspond to the labels in Fig. 14. 

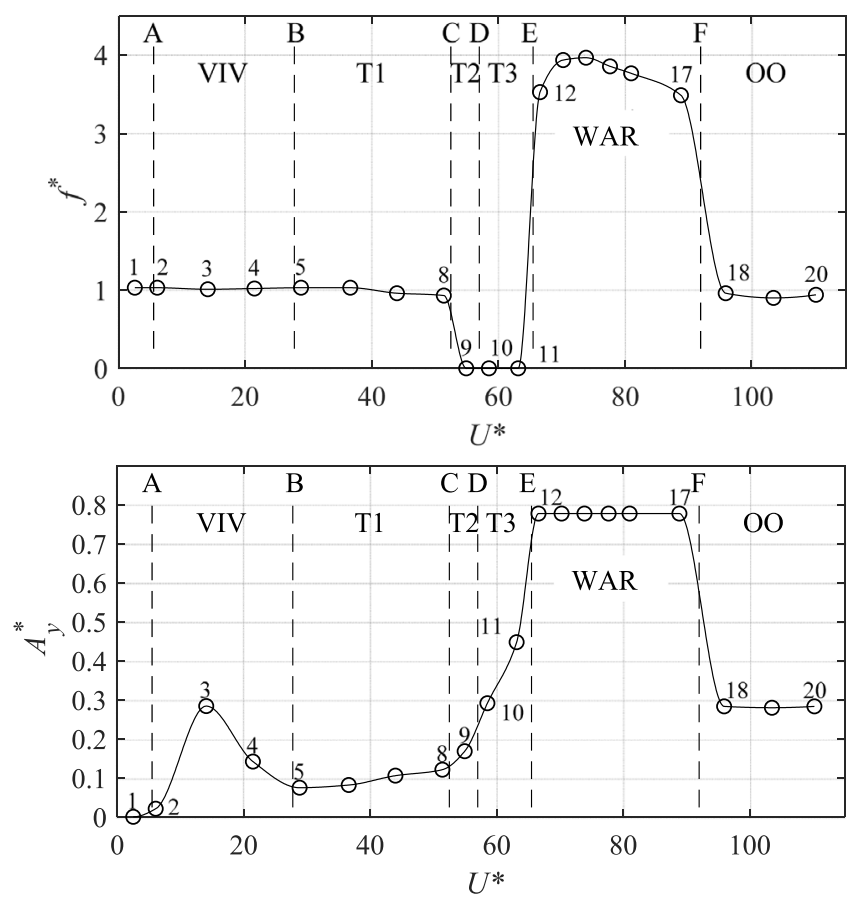

Fig. 14. Variation of the dimensionless amplitude $A_{y}^{*}$ and dimensionless frequency $f^{*}$ as a function of the reduced flow speed $U^{*}$. The experimental data are labeled from 1 to 20 . Letters A to F denote the limits of the different patterns (see Table 5). Points with $f^{*}=0$ : lack of a clear oscillation motion. WAR: Whirling AutoRotation.

of horizontal and vertical oscillation modes (of the same frequency and amplitude) in a plane transverse to the line direction is equivalent to a whirling motion (for modes whose associated centrifuge acceleration compensates gravity). This is one of the types of motion observed in the videos recorded in the field.

Frequencies and mode shapes derived from the elastic model and those from the video sequences are in agreement. The frequencies of aerodynamic loads that appear at moderate wind speeds generated by vortex shedding are in this same range, which suggests that this mechanism can be responsible for some kind of coupling between the system oscillations and the aerodynamic loads.

However, a difficulty arises when trying to explain the case of the autorotation motion contained in the transverse plane, from the aerodynamic point of view. Several instabilities can be explained by the changes of the aerodynamic forces along a cycle in which the body moves backward and forward in an incoming flow, in an oscillatory motion, leading to a change in the relative flow speed approaching the body (Jones, 1992). In the TILoW oscillatory case, there is no such a change in the relative incoming flow speed that could explain the instability. Vortex shedding could be a suitable explanation for oscillations (as in the case of a tethered sphere). In the TILoW autorotation case, the fluid flow can be considered at a local range as that of a translation motion, as the wind velocity relative to the beacon is steady as seen by a local observer (there is no oscillation anymore); the aerodynamic loads that sustain this steady autorotation motion are still to be explained.

In an attempt to reproduce this instability in the lab, wind tunnel tests have been performed using a simplified model, one cable with just one beacon (swinging sphere). In addition to the unperturbed case, another six motion patterns have been identified: oscillation synchronized VIV; whirling autorotation; three different behaviors in the transition between VIV and autorotation; and finally, the residual motion that appears when the autorotation disappears as the flow speed is increased.

The transitions from oscillation motion to autorotation, and the way the associated aerodynamic forces change, are both challenging research objectives.

\section{Acknowledgment}

We gratefully acknowledge the support and field data on overhead high tension lines provided by SAPREM.

\section{References}


Auton, T.R., 1987. The lift force on a spherical body in a rotational flow. J. Fluid Mech. 183, 199-218.

Bartoli, G., Cluni, F., Gusella, V., Procino, L., 2006. Dynamics of cable under wind action: wind tunnel experimental analysis. J. Wind Eng. Ind. Aerodyn. 94, 259-273.

Batill, S.M., Nebres, J.V.N., 1992. Detailed near surface flow about yawed, stranded cables. Contractor Report, CSS CR 1210-92-1, Naval Coastal Systems Center CR 2210-89-1.

Batill, S., Nelson, R., Nebres, J., 1989. An experimental investigation of the flow field around yawed stranded cables. Naval Coastal Systems Center CR 2210-89-1.

Carassale, L., Freda, A., Piccardo, G., 2005. Aeroelastic forces on yawed circular cylinders: quasi-steady modeling and aerodynamic instability. Wind Struct. 8, 373-388. http://dx.doi.org/10.12989/was.2005.8.5.373.

Chabart, O., Lilien, J.L., 1998. Galloping of electrical lines in wind tunnel facilities. J. Wind Eng. Ind. Aerodyn. 74-76, 967-976.

Cheng, S., Tanaka, H., 2005. Correlation of aerodynamic forces on an inclined circular cylinder. Wind Struct. 8 (2), 135-146. http://dx.doi.org/10.12989/was. 2005.8.2.135.

Cigada, A., Diana, G., Falco, M., Fossati, F., Manenti, A., 1997. Vortex shedding and wake-induced vibrations in single and bundle cables. J. Wind Eng. Ind. Aerodyn. 72, 253-263.

Dale, J., Menzel, H., McCandless, J., 1968. Dynamic characteristics of underwater cables flow induced transverse vibrations Report No. NADC-AE-6620.

Davis, D.A., Richards, D.J.W., Scriven, R.A., 1963. Investigation of conductor oscillation on the $275 \mathrm{kv}$ crossing over the rivers severn and wye. Proc. Inst. Electr. Eng. 110 (1), 205-219. http://dx.doi.org/10.1049/piee.1963.0028.

Eguchi, Y., Kikuchi, N., Kawabata, K., Yukino, T., Ishikubo, Y., 2002. Drag reduction mechanism and aerodynamic characteristics of a newly developed overhead electric wire. J. Wind Eng. Ind. Aerodyn. 90, 293-304.

EPRI, 2009. Transmission Line Reference Book-Wind-Induced Conductor Motion, Ed. Electric Power Research Institute, report no. 010185542009.

Govardhan, R.N., Williamson, C.H.K., 2005. Vortex-induced vibrations of a sphere. J. Fluid Mech. 531, 11-47. http://dx.doi.org/10.1017/S0022112005003757.

Horton, K.J., Ferrer, C.M., Watson, K.P.D., 1988. Measurements of the hydrodynamic force and strum characteristics of stranded cables, NCSC TM 471-87.

Hung, P.V., Yamaguchi, H., 2014. Extensive analyses on large amplitude gusty-wind induced vibrations of overhead transmission line systems. Research Report of Department of Civil and Environmental Engineering, Saitama Univ., 40, 46-56.

ICAO (International Civil Aviation Organization), 2009. Annex 14 Volume I, Aerodrome Design and Operations, Recommendation 6.2.9.

Jauvtis, N., Govardhan, R., Williamson, C.H.K., 2001. Multiple modes of vortex-induced vibration of a sphere. J. Fluids Struct. 15, 555-563. http://dx.doi.org/ $10.1006 / \mathrm{j} \# \mathrm{~s} .2000 .0348$.

Jones, K.F., 1992. Coupled vertical and horizontal galloping. ASCE J. Eng. Mech. 118 (1), 92-107, http://ascelibrary.org/doi/abs/10.1061/(ASCE)0733-9399.

King, R., 1976. Wake interaction experiments with two flexible circular cylinders in flowing water. J. Sound Vib. 45, 259-283. http://dx.doi.org/10.1016/ 0022-460X(76)90601-5.

Knisely, C.W., Kawagoe, M., 1990. Force-displacement measurements on closely spaced tandem cylinders. J. Wind Eng. Ind. Aerodyn. 33, 81-90. http: //dx.doi.org/10.1016/0167-6105(90)90023-6.

Macdonald, J.H.G., Griffiths, P.J., Curry, B.P., 2008. Galloping analysis of stranded electricity conductors in skew winds. Wind Struct. 11, 303-321. http: //dx.doi.org/10.12989/was.2008.11.4.303.

Macdonald, J.H.G., Larose, G.L., 2006. A unified approach to aerodynamic damping and drag/lift instabilities, and its application to dry inclined cable galloping. J. Fluids Struct. 22, 229-252.

Macdonald, J.H.G., Larose, G.L., 2008a. Two-degree-of-freedom inclined cable galloping-Part 2: Analysis and prevention for arbitrary frequency ratio. J. Wind Eng. Ind. Aerodyn. 96, 308-326.

Macdonald, J.H.G., Larose, G.L., 2008b. Two-degree-of-freedom inclined cable galloping- Part 1: General formulation and solution for perfectly tuned system. J. Wind Eng. Ind. Aerodyn. 96, 291-307.

Meirovitch, L., 2001. Fundamentals of Vibrations. McGraw-Hill.

Moraga, F.J., Bonetto, F.J., Lahey, R.T., 1999. Lateral forces on spheres in turbulent uniform shear flow. Int. J. Multiph. Flow. 25, $1321-1372$.

Nebres, J., Batill, S., 1993. Flow about a circular cylinder with a single large-scale surface perturbation. Exp. Fluids 15, 369-379. http://dx.doi.org/10.1007/ BF00191775.

Nebres, J., Batill, S., Nelson, R., 1993. Flow about yawed, stranded cables. Exp. Fluids 14, 49. http://dx.doi.org/10.1007/BF00196987.

Norman, A.K., Kerrigan, E.C., McKeon, B.J., 2011b. The effect of small amplitude time-dependent changes to the surface morphology of a sphere. J. Fluid Mech. 675, 268-296. http://dx.doi.org/10.1017/S0022112011000164.

Norman, A.K., McKeon, B.J, 2011a. The effect of a small isolated roughness element on the forces on a sphere in uniform flow. Exp. Fluids 51, $1031-1045$. http://dx.doi.org/10.1007/s00348-011-1126-y.

Provansal, M., Schouveiler, L., Leweke, T., 2004. From the double vortex street behind a cylinder to the wake of a sphere. Eur. J. Mech. B Fluids $23,65-80$.

Ramberg, S.E., 1978. The Influence of Yaw Angle Upon the Vortex Wakes of Stationary and Vibrating Cylinders, NRL Memorandum Report 3822.

Ruscheweyh, H.P., 1983. Aeroelastic interference effects between slender structures. J. Wind Eng. Ind. Aerodyn. 14, 129-140. http://dx.doi.org/10.1016/ 0167-6105(83)90017-X.

Shiraishi, N., 1986. On aerodynamic instabilities of tandem structures. J. Wind Eng. Ind. Aerodyn. 23, 437-447. http://dx.doi.org/10.1016/0167-6105(86) 90061-9.

Simpson, A., 1965. Aerodynamic instability of long-span transmission lines. Proc. Inst. Electr. Eng. 112, 315-324. http://dx.doi.org/10.1049/piee.1965.0050. Smith, B.W., 1993. A review of dynamic aspects of transmission line design. Eng. Struct. 15, 271-275. http://dx.doi.org/10.1016/0141-0296(93)90030-8.

von Kármán, T., 1948. Progress in the Statistical Theory of Turbulence. Proc. National Academy of Sciences, Washington, DC, pp. 530-539.

Wardlaw, R.L., Cooper, K.R., Ko, K.R., Watts, J.A., 1975. Wind tunnel and analytical investigations into the aeroelastic behaviour of bundled conductors. IEEE-PES Summer Meeting and Energy Resources Conf. Anaheim. http://dx.doi.org/10.1109/T-PAS.1975.31892.

Williamson, C.H.K., Govardhan, R., 1997. Dynamics and forcing of a tethered sphere in a fluid flow. J. Fluids Struct. 11, $293-305$.

Williamson, C.H.K., Govardhan, R., 2008. A brief review of recent results in vortex-induced vibrations. J. Wind Eng. Ind. Aerodyn. $96,713-735$.

Wong, H.Y., 1980. Vortex-induced wake buffeting and its suppression. J. Wind Eng. Ind. Aerodyn. 6, 49-57. http://dx.doi.org/10.1016/0167-6105(80)900215 .

Zdravkovich, M.M., 1977. Review of flow interference between two circular cylinders in various arrangements. J. Fluids Eng. 99, 618-633. http://dx.doi.org/ $10.1115 / 1.3448871$.

Zhou, C., Liu, Y., 2015. A theoretical model of rain-wind-induced in-plane galloping on overhead transmission tower-lines system. Adv. Mech. Eng. 7 (9), 1-10. http://dx.doi.org/10.1177/1687814015604590. 